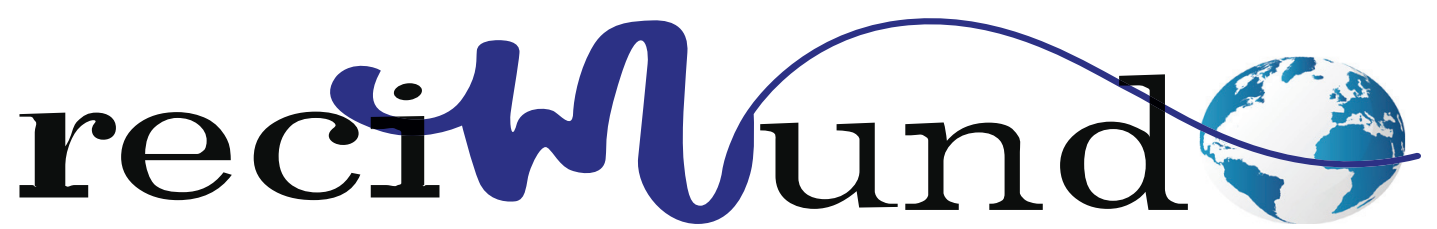

Revista Científica Mundo de la Investigación y el Conocimiento

DOI: 10.26820/recimundo/4.(4).octubre.2020.548-562

URL: http://recimundo.com/index.php/es/article/view/974

EDITORIAL: Saberes del Conocimiento

REVISTA: RECIMUNDO

ISSN: 2588-073X

TIPO DE INVESTIGACIÓN: Artículo de Revisión

CóDIGO UNESCO: 5909.01 Gestión Administrativa

PAGINAS: 548-562

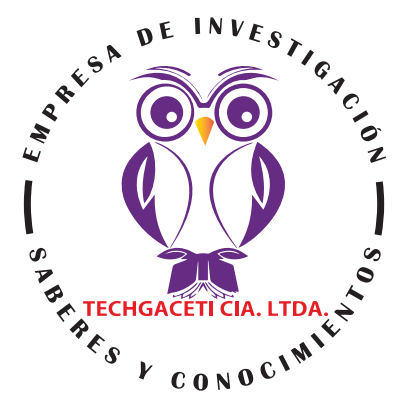

\title{
Calidad de los servicios en línea del GADM Chone
}

Quality of GADM Chone's online services

Qualidade dos serviços em linha do GADM Chone

Miryan Johanna Zambrano Zambrano1; Lucía Rivadeneira Barreiro²

\section{RECIBIDO: 19/09/2020 ACEPTADO: 21/09/2020 PUBLICADO: 15/10/2020}

1. Magister en Administración de Empresas Mención en Gerencia de la Calidad y Productividad; Ingeniera Comercial; Licenciada en Informática; Maestrante de la Pontificia Universidad Católica del Ecuador; Manabí, Ecuador; mzambrano@pucesm.edu.ec; https://orcid.org/0000-0003-2752-7649

2. Doctor of Philosophy in Business and Management; Master of Science (Information Systems); Ingeniero en Sistemas Informáticos; Licenciada en Ciencias de la Educación Mención Inglés; Pontificia Universidad Católica del Ecuador; Manabí, Ecuador; Irivadeneira@pucesm.edu.ec; https://orcid. org/0000-0001-5989-7703

CORRESPONDENCIA

Miryan Johanna Zambrano Zambrano

mzambrano@pucesm.edu.ec

Manabí, Ecuador 


\section{RESUMEN}

Este estudio tiene como finalidad analizar la calidad de los servicios en línea que el Gobierno Autónomo Descentralizado Municipal del cantón Chone (GADM Chone) brinda a los usuarios, en función de los criterios de diseño, accesibilidad y contenido. Los antecedentes determinan que los servicios en línea responden a una competencia directa del gobierno local contenida en el Código Orgánico de Organización Territorial, Autonomía y Descentralización (COOTAD) y conforme disposición de la Ley Orgánica de Transparencia y Acceso a la Información Pública (LOTAIP). El sustento teórico del presente estudio responde a la revisión y análisis de estudios indexados y científicos. El estudio de campo responde a los resultados obtenidos en la indagación de los criterios propuestos por expertos en diferentes áreas. El estudio muestra la estructura tecnológica del servicio municipal del GADM Chone y analiza cómo la innovación brinda facilidades a los usuarios por medio de la página web. Este aspecto es relevante sobre todo en los actuales momentos, en el que debido a la pandemia del Covid-19, las instituciones se han visto obligadas a disminuir el aforo en los espacios públicos, por lo que el acceso de los servicios en línea ha incrementado durante los últimos meses. En el caso de Chone, se refleja en el incremento del uso de servicios en línea por parte de los usuarios, puesto que cuenta con 30949 nuevos usuarios entre el 1 de enero y el 27 de agosto del 2020.

Palabras clave: Calidad, servicios en línea, página web, usuarios, GAD.

\section{ABSTRACT}

The purpose of this study is to analyze the quality of Chone's municipality's (GADM Chone) online services that is provided to users, based on design, accessibility and content criteria. The normative determines that online services are responsibility of the local government contained in the Organic Code of Territorial Organization, Autonomy and Decentralization (COOTAD) and in accordance with the provisions of the Organic Law of Transparency and Access to Public Information (LOTAIP). For the theoretical background, we analyzed indexed and academic journal articles. And, for the fieldwork, we included information from experts in different areas. The study shows the technological structure of the GADM Chone's online services and also analyzes how innovation supports users through its website. This aspect is relevant, especially in the current moments, in which due to the Covid-19 pandemic, institutions have been forced to reduce the capacity in public spaces, so access to online services has increased during the last months. In the case of Chone, it is evident in the increase of users of online services, since it has 30,949 new users between January 1 and August 27, 2020.

Keywords: Quality, online services, webpage, users, municipality.

\section{RESUMO}

O objectivo deste estudo é analisar a qualidade do serviço que a página web do município de Chone (GADM Chone) fornece aos utilizadores, com base nos critérios de concepção, acessibilidade e conteúdo. A normativa determina que os serviços online respondem a uma competência directa do governo local contida no Código Orgânico de Organização, Autonomia e Descentralização do Território (COOTAD) e em conformidade com as disposições da Lei Orgânica de Transparência e Acesso à Informação Pública (LOTAIP). O apoio teórico deste estudo responde à revisão e análise de estudos indexados e científicos. O estudo de campo provém dos critérios propostos por peritos em diferentes áreas. O estudo mostra a estrutura tecnológica dos serviços em linha do município de Chone e analisa a forma como a inovação apoia os utilizadores através do website. Este aspecto é relevante especialmente nos momentos actuais, em que devido à pandemia de Covid-19, as instituições foram forçadas a reduzir a capacidade nos espaços públicos, pelo que o acesso aos serviços em linha aumentou durante os últimos meses. No caso do Chone, é evidente no aumento de utilizadores de serviços em linha, uma vez que tem 30.949 novos utilizadores entre 1 de Janeiro e 27 de Agosto de 2020.

Palavras-chave: Qualidade, serviços online, página web, utilizadores, município. 


\section{INTRODUCCIÓN}

Actualmente, debido a la globalización, un sinnúmero de sociedades vive en la era digital, la cual está caracterizada por el acceso a la información a través de canales digitales. En este tipo de sociedades, los servicios virtuales, llamados también servicios en línea, pasaron de ser una opción a una necesidad, puesto que se convirtieron en un imperativo para las grandes mayorías en el desarrollo de sus actividades. Los servicios en línea son aquellos servicios que son brindados a través de Internet.

En este sentido, la comunicación realizada a través de los servicios en línea entre las organizaciones y los usuarios es un aspecto relevante y vigente durante los últimos diez años. Dicha comunicación, que puede manifestarse a través de los servicios en línea, se ha venido innovando constantemente en función de las demandas, de las opciones que ofrece el vertiginoso avance de la tecnología y de la capacidad de respuesta de las organizaciones, sean éstas, públicas o privadas.

La presente investigación es una mirada analítica para determinar la calidad de los servicios en línea que el Gobierno Autónomo Descentralizado Municipal del cantón Chone (GADM) en Ecuador, ha establecido para permitir el acceso de los usuarios a la información y servicios públicos que se ofrecen en línea a través de su página web. Para este estudio, la calidad es definida como la capacidad de un servicio determinado para motivar y satisfacer las necesidades de los destinatarios o usuarios (Zambrano Paladines et al, 2019). Los servicios en línea que se pueden ofrecer a través de las páginas web municipales son todos aquellos que no requieran la presencia del usuario, estos pueden ser información, consultas, trámites básicos, agendamiento de citas, pagos, impresión de certificados o mecanizados, entre otros. La finalidad de estos servicios en línea es reducir los tiempos de respues- tas y optimizar los recursos, tanto de la entidad municipal como de los usuarios. Esta optimización de recursos, favorece la institucionalidad y el desarrollo personal de los ciudadanos, tal cual lo determina el Código Orgánico de Organización Territorial, Autonomía y Descentralización (COOTAD) en su artículo 28 que indica que "cada circunscripción territorial tendrá un gobierno autónomo descentralizado para la promoción del desarrollo y la garantía del buen vivir, a través del ejercicio de sus competencias" (Asamblea, 2010).

En concordancia, el GADM Chone conforme sus competencias normativas establecidas en el artículo 29 del COOTAD y desde la emisión de esta norma, creó su página web institucional. Inicialmente, la misma fue diseñada como fuente de información y posteriormente se la modificó para transparentar la gestión de la institución, conforme lo dispone el artículo 7 de la Ley Orgánica de Transparencia y Acceso a la Información Pública (LOTAIP) (Congreso, 2004). Actualmente, se han implementado el acceso a servicios en línea que están orientados mayormente al desarrollo de trámites burocráticos, que en el pasado eran llevado a cabo sólo de forma presencial.

El período de evolución de los servicios en línea del GADM Chone provienen del año 2010. A partir de este año se creó la página web con la función informativa, es decir, para ofrecer información acerca de la composición institucional de sus autoridades y para comunicar las actividades que desarrollaba la institución. Posteriormente, se incorporaron los servicios de transparencia según lo determina la LOTAIP, en donde se informa a la ciudadanía la planificación por período administrativo, la rendición de cuentas mensual del cumplimiento de esa planificación por departamentos, así como las actuaciones del Concejo respecto a las sesiones y ordenamiento jurídico local aprobado. Sin embargo, desde hace seis años, por disposición del Concejo de Participa- 
ción Ciudadana y Control Social (CPCCS), se publican también dentro de los servicios de información de transparencia los procesos de contratación pública llevados a cabo por la institución desde

que inicia la etapa precontractual hasta la finalización, a través de adjudicaciones o resoluciones de procesos desiertos. A partir del 2015, se incorporó el acceso a servicios en línea, comprendido mayormente por trámites como el pago de tributos y servicios básicos, o certificaciones de patentes para gestiones complementarias en otras instituciones públicas y privadas, los mismos que son parte de las demandas ciudadanas, cuya implementación en línea da celeridad al servicio.

El GADM Chone tiene competencia administrativa, geográfica y política en las siete parroquias rurales que tienen igual número de instituciones responsables de la administración territorial, y en dos parroquias urbanas que componen su cabecera cantonal. A los habitantes de estas parroquias se les ha hecho conocer de la existencia de los servicios en línea a través de la página web institucional. Estos servicios han sido ofertados como procesos a los que se pueden acceder de manera sencilla, mismos que permiten la conexión directa entre la institución y la ciudadanía para desarrollar trámites que en el pasado ocupaban más recursos, especialmente en cuestión de tiempo. $\mathrm{Si}$ bien los servicios en línea del GADM Chone han estado disponibles y funcionales desde hace 5 años, éstos no han sido ampliamente utilizados, puesto que los usuarios prefieren llevar a cabo los trámites burocráticos de forma presencial. Sin embargo, el panorama actual ha cambiado debido a la pandemia del Covid-19 ocurrida a finales del primer trimestre del 2020, en donde instituciones públicas y privadas se han visto en la necesidad de cerrar la atención física a usuarios y trasladar sus servicios en línea. En el caso del GADM Chone, los ciudadanos se vieron obligados a usar estos servicios con la finalidad de cumplir con sus obligaciones como el pago de tributos o de servicios, así como para obtener certificaciones de avalúos o no deudor. Por este motivo, este estudio tiene como objetivo determinar la calidad de los servicios en línea en el GADM Chone, para lo cual se hizo necesario, primero identificar los servicios que ofrece la página web municipal y los trámites que se pueden realizar a través de ella. En base a esa información se identifican los criterios de evaluación de la calidad de los servicios en línea del GADM Chone, disponibles en su página web. Estos criterios son resultado del análisis de expertos en diferentes áreas.

El resto del artículo está organizado en cuatro secciones: la Sección 2 presenta la revisión de literatura donde se introducen los estudios relevantes que dan soporte a esta investigación. La Sección 3 detalla la metodología usada para la indagación y la Sección 4 muestra los resultados. Finalmente, la conclusión es presentada en la Sección 5.

\section{REVISION DE LITERATURA}

El sustento teórico del presente estudio se fundamenta en estudios paralelos como el desarrollado por Díaz (2018) para determinar la funcionalidad de la página y sus posibles vulnerabilidades, el de Paladines (2015) para determinar el cumplimiento de la LOTAIP en un municipio de Ecuador, y el de Sánchez y Villarejo (2004) para proponer una herramienta de diagnóstico de la calidad de servicio electrónico. Estos estudios reflejan cómo los servicios en línea se pueden convertir en una herramienta eficiente para que los gobiernos centrales y locales brinden a los ciudadanos atención de calidad. Los beneficios, tanto para las entidades gubernamentales como para los usuarios suelen ser múltiples, como por ejemplo la optimización de los procesos que conlleva el ahorro del tiempo y recursos financieros, materiales y de logística institucional.

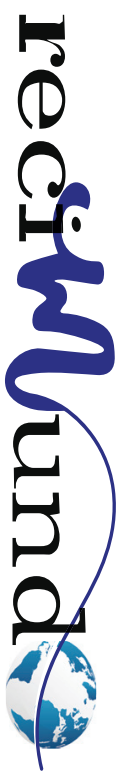


Otro de los beneficios es la disponibilidad de la información sin horarios o distancias, ya que los usuarios pueden consultar en el momento que requieran el servicio.

Las páginas web son portales digitales que permiten mostrar, enviar y recibir información. Estos portales permiten la interacción entre instituciones y usuarios. Ante este panorama surge la pregunta de cuál debe ser la estructura de una página web

municipal y qué servicios en línea puede ofrecer. En respuesta a la primera parte de la pregunta, la empresa PentaWeb, orientada a ofrecer diseños web profesionales, sugiere que las páginas digitales deben contener tres elementos básicos que son cabecera, cuerpo y pie. Estos elementos se refieren a la graficación de las secciones en el portal, al contenido y al cierre, mismo que por lo general contiene contactos, información legal, política de privacidad, cookies y condiciones de uso, entre otros. También indica que los portales deben estructurarse en un esquema de cuatro secciones que son el área de Inicio o Home, que contiene información general de la institución, productos o servicios donde se publicita los bienes o servicios ofrecidos, blog que contiene la información institucional, y contactos que son los números de teléfonos y correos electrónicos a los que los usuarios se pueden comunicar con la organización (PentaWeb, 2017 ). En la misma línea, otra empresa de soluciones digitales llamada Incdustry sugiere que las páginas web deben contener cinco secciones, siendo estas ¿Quiénes somos? orientada a brindar información acerca de la institución, productos o servicios, el portafolio que enumera los servicios que ofrece la organización, blog y contacto (Incdustry, 2019). Finalmente, una tercera empresa dedicada a la actividad de servicios en línea llamada Masdigital recomienda que las secciones debieran ser seis: Productos o servicios, blog, contacto, pago o tienda online, preguntas frecuentes y galería de fotos y videos (Masdigital, 2016).
Las tres empresas nombradas anteriormente coinciden en que hay tres criterios que tienen que ser utilizados para medir la calidad del servicio en línea. Estos son diseño, accesibilidad y contenido.

El diseño de una página web es el primer punto de acceso de un usuario. Éste contempla aspectos como los colores, tipo de letra, logotipo y fotografías o vídeos que se convierten en los conectores con la institución. Al respecto, Hernández (2018) sostiene que el tiempo del que dispone una organización para captar un usuario es de muy pocos segundos, por lo tanto, la primera impresión que tienen los usuarios al acceder a una página web es en algunos casos definitiva para determinar su posterior utilización. Por lo que, se puede determinar que el diseño, es la carta de presentación de un servicio de calidad.

La accesibilidad no sólo depende del servidor de Internet que tenga la organización o el usuario a su disponibilidad. Es también la sincronización que tenga la presentación de una página web, y que de manera simple oriente a los usuarios para facilitar su uso. Al respecto, Berners-Le (2019) afirma que tanto para las personas involucradas en el desarrollo de las páginas web, como para quienes la acceden, la accesibilidad debe ser la herramienta vital de la calidad del producto o servicio que busca ofrecer.

El contenido de la página web tiene dos componentes, lo que la organización quiere ofrecer y lo que el usuario necesita adquirir. En este sentido, no toda la información que tiene una página web es de interés del beneficiario, pero en todo momento debe haber un hilo conector para que el servicio en línea cumpla con los estándares de calidad que faculta la tecnología. Merlo (2013) sostiene que el contenido de una página web más relevante está relacionado con la combinación de información hipertextual y multimedia, acompañada de información concreta y concisa a disposición de los usuarios. 
En su conjunto, la calidad del servicio web depende de estos tres componentes, así como la facilidad de navegación y características técnicas, tales como la capacidad del servidor de servicios de Internet o de las computadoras desde donde se acceden a los servicios. Lípeez-De Castro y García Alonso (2016) señalan algunos aspectos relevantes para identificar la provisión de información, consulta, el compromiso y la participación ciudadana. Este estudio considera necesaria la presentación del Plan de Desarrollo Territorial que es la planificación de largo plazo, la publicación de ordenanzas y

resoluciones municipales, Plan Operativo Anual que contiene la planificación anual con recursos y responsables, informe de rendición de cuentas, tal cual lo determina la LOTAIP (Congreso, 2004).

El estudio llevado a cabo por Zambrano et al. (2019), se orientó a analizar y evaluar cinco aspectos de los portales web de los GADM del Ecuador. Estos son: información, interacción, transacción, integración y participación. Este estudio concluye que la información es la que mayor cumplimiento refleja entre las páginas web, ya que el $71 \%$ de los gobiernos municipales y provinciales tiene información completa y actualizada de los servicios que ofrecen las entidades municipales (Zambrano et al., 2019). Al respecto de información, Jarrín (2017) sostiene que la tecnología influye en la satisfacción de los usuarios, puesto que representa obtener el mismo servicio, que tradicionalmente ha sido realizado de manera física, de forma ágil a través de la implementación de los servicios en línea, lo que representa un ahorro de recursos.

La gobernanza global debe innovarse en todos los aspectos. Esto es lo que sugiere Sanahuja (2020) quién realizó un análisis de la respuesta tecnológica de las organizaciones frente a los desafíos actuales en aspectos como economía y resiliencia. Sanahuja
(2020) indica también que es imprescindible incorporar todo conocimiento experto al ejercicio de las políticas públicas, mismo que tiene relación con la implementación y uso de los servicios en línea institucionales.

\section{METODOLOGÍA}

Este estudio es exploratorio y surgió del cuestionamiento de la calidad de servicios en línea del GADM Chone, la investigación parte de un análisis documental que ayudó a identificar los criterios para el análisis de la página web del GADM Chone, conforme lo estipulado en el COOTAD Y LOTAIP. Posteriormente, se analizó el criterio de tres empresas de diseño web que son PentaWeb, Incdustry y Masdigital, las que sugieren tres criterios para análisis que son diseño, accesibilidad y contenido, las que a su vez cuentan con criterios específicos para su calificación.

Para definir los indicadores y aspectos de calidad a evaluar se aplicó una herramienta cualitativa denominada criterio de expertos. Esta herramienta es una matriz de valoración especializada que contó con el aporte académico de profesionales en temas relacionados a la calidad de los servicios en línea, provenientes de dos universidades locales, que son la Universidad Laica Eloy Alfaro (ULEAM) y la Universidad Técnica de Manabí (UTM). Se consultó además con tres expertos en programación quienes definieron las características de programación; un comunicador social que definió las pautas para el contenido e información; dos representantes de asociaciones barriales y de comerciantes para conocer las expectativas en torno al servicio en línea; un diseñador gráfico quien determinó los criterios técnicos de imagen de una página web, y finalmente se consultó a un abogado experto en la normativa legal del COOTAD y la LOTAIP, quien puntualizó los términos legales que deben cumplir los GADM municipales referente a la información y comunicación establecidas en la Ley. 
Con los resultados de esta técnica se determinaron los criterios de evaluación e indicadores de calidad, que permitieron el diseño de una matriz para registrar, ponderar y evaluar los componentes de la página web del GADM Chone, para medir la calidad de sus servicios en línea.

\section{RESULTADOS}

El resultado está constituido por el diagnóstico realizado a la página web del GADM Chone y fue proporcionado por funcionarios de dicha entidad. Según los datos proporcionados, se evidencia 30.949 nuevos usuarios entre el 1 de enero y el 27 de agosto

del 2020, como se visualiza en la Figura 1. El total de usuarios, accede a los servicios en línea del GADM Chone desde Ecuador constituyendo el 86,16\% del total, seguido por residentes en Estados Unidos en un 6,77\%, y en menor proporción residentes ecuatorianos en Europa y América Latina. La mayor demanda del servicio en línea se registró en el mes de junio del 2020, tal como se refleja en la Figura 2, y la mayor cantidad de visitas se realizó desde la provincia de Manabí constituyendo el 50,77\% del total, seguido de la provincia del Guayas con $17,73 \%$ y Pichincha con $16,61 \%$ tal como se visualiza en la Figura 3 (GADM Chone, 2020).

\begin{tabular}{|c|c|c|}
\hline Pais & Usuarios & \% Usuarios \\
\hline 1. Ecuador & 27.502 & $86,18 \%$ \\
\hline 2. . United States & 2.160 & | $6,77 \%$ \\
\hline 3. Russia & 430 & $1,35 \%$ \\
\hline 4. I Spain & 326 & $1,02 \%$ \\
\hline 5. $=$ Colombia & 207 & $0,65 \%$ \\
\hline 6. III Peru & 185 & $0,58 \%$ \\
\hline 7. UII Mexico & 157 & $0,49 \%$ \\
\hline 8. = Argentina & 134 & $0,42 \%$ \\
\hline 9. (not set) & 84 & $0,26 \%$ \\
\hline 10. L Chile & 71 & $0,22 \%$ \\
\hline
\end{tabular}

Figura 1. Proporción de usuarios que acceden a los servicios en línea del GADM Chone, según el país del que se accede.

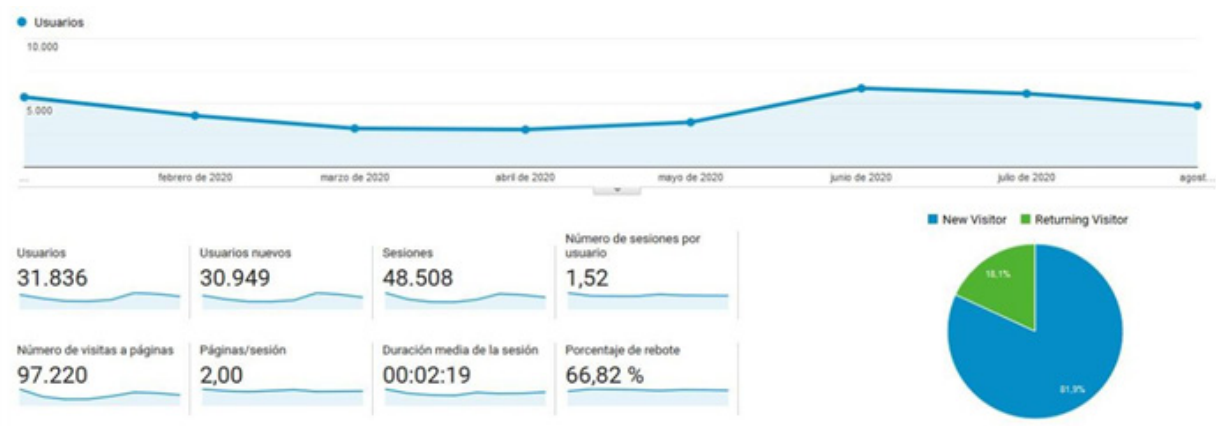

Figura 2. Proporción mensual de la demanda de los servicios en línea del GADM Chone. 


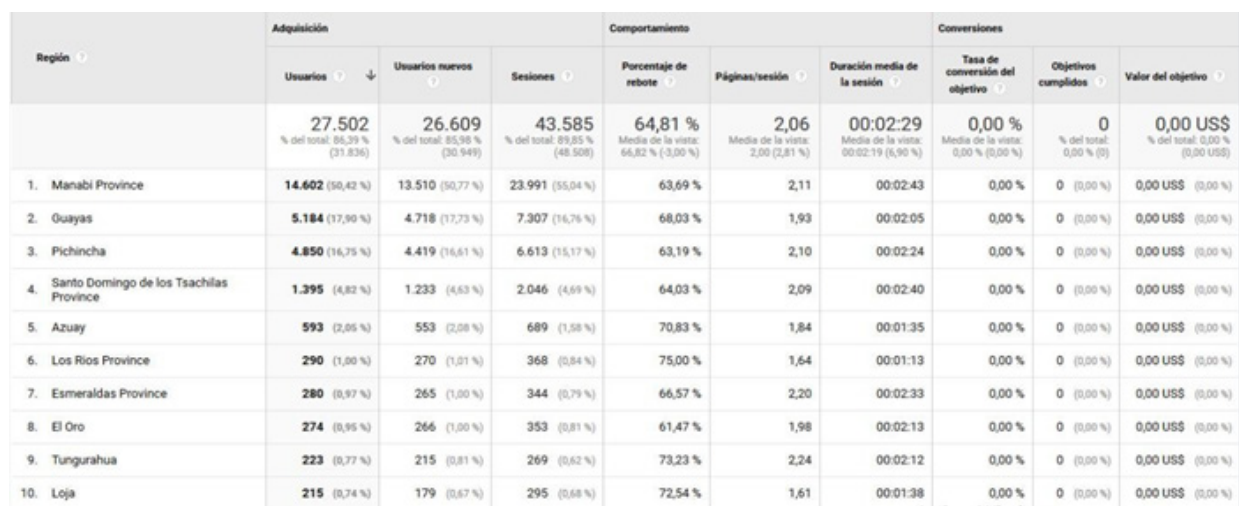

Figura 3. Proporción de visitas al GADM Chone de acuerdo a las provincias del Ecuador.

Los criterios establecidos por los expertos fueron divididos en tres secciones: diseño, accesibilidad y contenido. Para este estudio, el contenido está conformado por: información, servicios y productos, y consultas y trámites como se aprecia en la Figura 4. El porcentaje asignado en el aporte de las variables de estudio fue determinado en base al criterio de expertos, como se indicó en la metodología.

\begin{tabular}{|c|c|c|}
\hline & VARIABLES DE ESTUDIO & APORTE \\
\hline \multirow[b]{4}{*}{ Contenido } & $\begin{array}{l}\text { 1. Diseño de la página web del } \\
\text { GAD Municipal de Chone }\end{array}$ & $15 \%$ \\
\hline & $\begin{array}{l}\text { 2. Accesibilidad de la página web } \\
\text { del GAD Municipal de Chone }\end{array}$ & $15 \%$ \\
\hline & 3. Información & $30 \%$ \\
\hline & 4. Servicios y productos & $25 \%$ \\
\hline & 5. Consultas y trámites & $15 \%$ \\
\hline
\end{tabular}

Figura 4. Criterios establecidos para evaluar la calidad de los servicios en línea del GADM Chone.

El diseño de la página web del GADM Chone cumple con todos los ítems de evaluación, tal como se aprecia en la Tabla 1. Las valoraciones dadas responden al análisis del porcentaje de cumplimiento técnico de los ítems y este criterio tributa en un 15\% al resultado general. En el caso de Chone su valoración total es del $85 \%$ lo cual representa un $12,73 \%$. En este contexto, se determina que la página web consta de un diseño amigable y cumple con los parámetros establecidos, según la siguiente escala valorativa: 1 Muy malo, 2 Malo, 3 Regular, 4 Bueno, 5 Muy bueno. 
Tabla 1. Análisis del diseño de la página web del GADM Chone

\begin{tabular}{|c|c|c|c|c|c|}
\hline \multirow{2}{*}{$\begin{array}{l}\text { 1. Diseño de la página web del GAD } \\
\text { Municipal de Chone }\end{array}$} & \multicolumn{5}{|c|}{ Valoración } \\
\hline & 1 & 2 & 3 & 4 & 5 \\
\hline 1.1 ¿Tiene encabezados claros? & & & & $\mathrm{x}$ & \\
\hline 1.2 ¿Cuenta con imágenes atractivas? & & & & $\mathrm{x}$ & \\
\hline 1.3 ¿La fuente de letra es clara? & & & & & $\mathrm{x}$ \\
\hline 1.4 ¿El tamaño de letra es adecuado? & & & & $\mathrm{x}$ & \\
\hline 1.5 ¿El diseño es dinámico? & & & & & $\mathrm{x}$ \\
\hline 1.6 ¿Los colores usados son amigables? & & & & $\mathrm{x}$ & \\
\hline 1.7 ¿El diseño anima a seguir en la página? & & & & $\mathrm{x}$ & \\
\hline $\begin{array}{c}1.8 \text { ¿La distribución de elementos es } \\
\text { amigable? }\end{array}$ & & & & $\mathrm{x}$ & \\
\hline Total & 0 & 0 & 0 & 24 & 10 \\
\hline SUMA DE TOTALES & \multicolumn{5}{|c|}{34} \\
\hline PORCENTAJE DE CUMPLIMIENTO & \multicolumn{5}{|c|}{$\mathbf{1 2 , 7 3 \%}$} \\
\hline APORTE AL RESULTADO GENERAL & \multicolumn{5}{c}{} \\
\hline
\end{tabular}

En el análisis de accesibilidad se consideraron nueve ítems de evaluación tal como se refleja en la Tabla 2. Este criterio aporta en un 15\% al resultado general y la valoración obtenida es del $84 \%$, lo cual representa un 12,67\%. La accesibilidad es evaluada desde la perspectiva de la navegación o acceso, sea esta desde un computador o un teléfono inteligente. En base a los resultados, se identifica que la página web es accesible desde cualquier artefacto que permita la navegación web y se determina además que la experiencia de navegación es amigable con el usuario, destacando la facilidad de su uso mediante las aplicaciones que posee. Sin embargo, el aspecto a mejorar es el tiempo de respuestas de los enlaces y secciones de la misma.

Tabla 2. Análisis de accesibilidad de la página web del GADM Chone

\begin{tabular}{|l|c|c|c|c|c|}
\hline \multirow{2}{*}{$\begin{array}{l}\text { 2. Accesibilidad de la página web del GAD } \\
\text { Municipal de Chone }\end{array}$} & \multicolumn{5}{|c|}{ Valoración } \\
\cline { 2 - 6 } & 1 & 2 & 3 & 4 & 5 \\
\hline 2.1 ¿La navegación es rápida? & & & & $\mathrm{x}$ & \\
\hline 2.2 ¿La navegación es fácil? & & & & $\mathrm{x}$ & \\
\hline 2.3 ¿Contiene enlaces de referencia o guía? & & & & $\mathrm{x}$ & \\
\hline 2.4 ¿El sistema es amigable? & & & $\mathrm{x}$ & & \\
\hline 2.5 ¿Brinda respuestas rápidas? & & & & & $\mathrm{x}$ \\
\hline $\begin{array}{c}2.6 \text { ¿Es posible la navegación desde un } \\
\text { smartphone? }\end{array}$ & & & & $\mathrm{x}$ & \\
\hline $\begin{array}{l}2.7 \text { ¿La navegación desde un smartphone es } \\
\text { amigable? }\end{array}$ & & & & & \\
\hline 2.8 ¿Cuenta con una aplicación para PC? & & & & & $\mathrm{x}$ \\
\hline
\end{tabular}


2.9 ¿Cuenta con una aplicación móvil para smartphone?

\begin{tabular}{|c|c|c|c|c|c|}
\hline IOIC. & & & & & \\
\hline Total & 0 & 0 & 3 & 20 & 15 \\
\hline SUMA DE TOTALES & \multicolumn{5}{|c|}{38} \\
\hline PORCENTAJE DE CUMPLIMIENTO & \multicolumn{5}{|c|}{$84 \%$} \\
\hline APORTE AL RESULTADO GENERAL & \multicolumn{5}{|c|}{$12,67 \%$} \\
\hline
\end{tabular}

En el criterio información de la sección de contenidos existen 16 ítems de evaluación (Tabla 3). El aporte al resultado general es del 30\% y la ponderación obtenida es del 91\%, lo cual representa un $27,34 \%$. Se evidencia además que tiene un porcentaje de cumplimiento de 93,75\% cumpliendo con 15 de los 16 ítems, exceptuando la información de los funcionarios. Se identifica que existe información de las autoridades, sin embargo, es limitada a una presentación del Concejo Cantonal y carece de información relacionada a contactos telefónicos u otros medios. Pese a esto, se determina que este criterio cumple satisfactoriamente los estándares en base a lo establecido en la LOTAIP y los indicadores determinados en el criterio de expertos.

Tabla 3. Análisis de información de la página web del GADM Chone

\begin{tabular}{|c|c|c|c|c|c|c|c|}
\hline \multirow{2}{*}{ 3. Información } & \multicolumn{2}{|c|}{\begin{tabular}{|c|} 
Cumplimient \\
$\mathrm{o}$
\end{tabular}} & \multicolumn{5}{|c|}{ Valoración } \\
\hline & $\mathrm{Si}$ & No & 1 & 2 & 3 & 4 & 5 \\
\hline 3.1 ¿Tiene información de la institución? & $\mathrm{x}$ & & & & & & $\mathrm{x}$ \\
\hline 3.2 ¿Esta publicada la Misión y Visión? & $\mathrm{x}$ & & & & & & $\mathrm{x}$ \\
\hline 3.3 ¿Tiene información de autoridades? & $\mathrm{x}$ & & & & $\mathrm{x}$ & & \\
\hline 3.4 ¿Tiene información de funcionarios? & & $\mathrm{x}$ & & & & & \\
\hline 3.5 ¿Se publican las ordenanzas? & $\mathrm{x}$ & & & & & & $\mathrm{x}$ \\
\hline 3.6 ¿Se publican las actas de sesión de concejo? & $\mathrm{x}$ & & & & & & $\mathrm{x}$ \\
\hline 3.7 ¿Esta publicado el PDOT cantonal? & $\mathrm{x}$ & & & & & & $\mathrm{x}$ \\
\hline 3.8 ¿Esta publicado el Plan Operativo Anual? & $\mathrm{x}$ & & & & & & $\mathrm{x}$ \\
\hline $\begin{array}{l}3.9 \text { ¿Están publicados los procesos de } \\
\text { contratación? }\end{array}$ & $\mathrm{x}$ & & & & & & $\mathrm{x}$ \\
\hline $\begin{array}{l}3.10 \text { ¿Están publicadas las adjudicaciones de } \\
\text { contrato? }\end{array}$ & $\mathrm{x}$ & & & & & & $\mathrm{x}$ \\
\hline $\begin{array}{l}3.11 \text { ¿Esta publicada la última rendición de } \\
\text { cuentas? }\end{array}$ & $\mathrm{x}$ & & & & & & $\mathrm{x}$ \\
\hline 3.12 ¿Están publicados los horarios de atención? & $\mathrm{x}$ & & & & & & $\mathrm{x}$ \\
\hline 3.13 ¿Está publicada información del cantón? & $\mathrm{x}$ & & & & & & $\mathrm{x}$ \\
\hline $\begin{array}{l}3.14 \text { ¿Están publicados los contactos de la } \\
\text { institución? }\end{array}$ & $\mathrm{X}$ & & & & & & $\mathrm{x}$ \\
\hline 3.15 ¿Cuenta con una sección de noticias? & $\mathrm{x}$ & & & & & & $\mathrm{x}$ \\
\hline 3.16 ¿Cuenta con un Blog? & $\mathrm{x}$ & & & & & & $\mathrm{x}$ \\
\hline \multicolumn{3}{|l|}{ Total } & 0 & 0 & 3 & 0 & 70 \\
\hline \multicolumn{3}{|l|}{ SUMA DE TOTALES } & \multicolumn{5}{|c|}{73} \\
\hline \multicolumn{3}{|l|}{ PORCENTAJE DE CUMPLIMIENTO } & \multicolumn{5}{|c|}{$91 \%$} \\
\hline \multicolumn{3}{|l|}{ APORTE AL RESULTADO GENERAL } & \multicolumn{5}{|c|}{$27,34 \%$} \\
\hline
\end{tabular}


El criterio servicios y productos de la sección de contenidos consta de 12 ítems de evaluación como se visualiza en la Tabla 4. El aporte al resultado general es del 25\% y la ponderación obtenida es del $83 \%$, lo que representa un 20,83\%. Se evidencia además que tiene un porcentaje de cumplimiento de 91,67\% cumpliendo con 11 de los 12 ítems que comprenden este criterio, con excepción del pago en línea de servicios de matriculación.

Tabla 4. Análisis de servicios y productos de la página web del GADM Chone

\begin{tabular}{|c|c|c|c|c|c|c|c|}
\hline \multirow{2}{*}{ 4. Servicios y productos } & \multicolumn{2}{|c|}{$\begin{array}{c}\text { Cumplimient } \\
\mathrm{o}\end{array}$} & \multicolumn{5}{|c|}{ Valoración } \\
\hline & $\mathrm{Si}$ & No & 1 & 2 & 3 & 4 & 5 \\
\hline 4.1 ¿Se pueden descargar mecanizados? & $\mathrm{x}$ & & & & & & $\mathrm{x}$ \\
\hline 4.2 ¿Se pueden descargar certificados? & $\mathrm{x}$ & & & & & & $\mathrm{x}$ \\
\hline 4.3 ¿Se pueden pagar los impuestos prediales? & $\mathrm{x}$ & & & & & & $\mathrm{x}$ \\
\hline 4.4 ¿Se pueden pagar los impuestos comerciales? & $\mathrm{x}$ & & & & & & $\mathrm{x}$ \\
\hline 4.5 ¿Se pueden pagar las patentes municipales? & $\mathrm{x}$ & & & & & & $\mathrm{x}$ \\
\hline 4.6 ¿Se puede pagar el servicio de agua potable? & $\mathrm{x}$ & & & & & & $\mathrm{x}$ \\
\hline 4.7 ¿Se puede pagar la matriculación vehicular? & & $\mathrm{x}$ & & & & & \\
\hline 4.8 ¿Se pueden pagar multas y/o sanciones? & $\mathrm{x}$ & & & & & & $\mathrm{x}$ \\
\hline $\begin{array}{l}4.9 \text { ¿Se pueden cancelar los cánones de } \\
\text { arrendamiento? }\end{array}$ & $\mathrm{x}$ & & & & & & $\mathrm{x}$ \\
\hline 4.10 ¿Se pueden realizar trámites en línea? & $\mathrm{x}$ & & & & $\mathrm{x}$ & & \\
\hline 4.11 ¿Se pueden agendar citas? & $\mathrm{x}$ & & & & $\mathrm{x}$ & & \\
\hline 4.12 ¿Se pueden realizar reclamos y sugerencia? & $\mathrm{x}$ & & & & & $\mathrm{x}$ & \\
\hline \multicolumn{3}{|l|}{ Total } & 0 & 0 & 6 & 4 & 40 \\
\hline \multicolumn{3}{|l|}{ SUMA DE TOTALES } & \multicolumn{5}{|c|}{50} \\
\hline \multicolumn{3}{|l|}{ PORCENTAJE DE CUMPLIMIENTO } & \multicolumn{5}{|c|}{$83 \%$} \\
\hline \multicolumn{3}{|l|}{ APORTE AL RESULTADO GENERAL } & \multicolumn{5}{|c|}{$20,83 \%$} \\
\hline
\end{tabular}

La Tabla 5 contiene los 10 ítems de evaluación correspondientes al criterio de consultas y trámites de la sección de contenidos. El aporte al resultado general es del 15\%, la ponderación obtenida por la página web del GADM Chone es del 36\%, esto representa un 5,41\%. En este criterio se puede evidenciar un cumplimiento del $40 \%$ debido a que no se evidencia guías para proveedores y contratistas, y para el llenado de pliegos. Tampoco permite darle seguimiento en tiempo real a los trámites, ni la interacción con las autoridades, o evaluar la gestión municipal. Se identifica además que la interacción con los funcionarios es limitada a correos electrónicos únicamente y el contacto con las áreas de la institución es telefónica con líneas de extensión. 
Tabla 5. Análisis de consultas y trámites de la página web del GADM Chone

\begin{tabular}{|c|c|c|c|c|c|c|c|}
\hline \multirow{2}{*}{ 5. Consultas y trámites } & \multicolumn{2}{|c|}{ Cumplimiento } & \multicolumn{5}{|c|}{ Valoración } \\
\hline & $\mathrm{Si}$ & No & 1 & 2 & 3 & 4 & 5 \\
\hline 5.1 ¿Permite realizar consultas? & $\mathrm{x}$ & & & & & & $\mathrm{x}$ \\
\hline 5.2 ¿Existe la opción de preguntas frecuentes? & $\mathrm{x}$ & & & & & & $\mathrm{x}$ \\
\hline 5.3 ¿Esta publicada una guía para trámites? & $\mathrm{x}$ & & & & & & $\mathrm{x}$ \\
\hline $\begin{array}{l}5.4 \text { ¿Esta publicada una guía para proveedores y } \\
\text { contratistas? }\end{array}$ & & $\mathrm{x}$ & & & & & \\
\hline 5.5 ¿Esta publicada una guía para llenar pliegos? & & $\mathrm{x}$ & & & & & \\
\hline 5.6 ¿Permite dar seguimiento a los trámites? & & $\mathrm{x}$ & & & & & \\
\hline 5.7 ¿Permite la interacción con autoridades? & & $\mathrm{x}$ & & & & & \\
\hline 5.8 ¿Permite la interacción con funcionarios? & $\mathrm{x}$ & & & & $\mathrm{x}$ & & \\
\hline 5.9 ¿Permite evaluar la gestión municipal? & & $\mathrm{x}$ & & & & & \\
\hline 5.10¿Permite evaluar la página Web? & & $\mathrm{x}$ & & & & & \\
\hline \multicolumn{3}{|l|}{ Total } & 0 & 0 & 3 & 0 & 15 \\
\hline \multicolumn{3}{|l|}{ SUMA DE TOTALES } & \multicolumn{5}{|c|}{18} \\
\hline \multicolumn{3}{|l|}{ PORCENTAJE DE CUMPLIMIENTO } & \multicolumn{5}{|c|}{$36 \%$} \\
\hline \multicolumn{3}{|l|}{ APORTE AL RESULTADO GENERAL } & \multicolumn{5}{|c|}{$5,41 \%$} \\
\hline
\end{tabular}

Los porcentajes de cumplimiento refieren a cumplir en alguna medida el ítem señalado. En este análisis no se ve en qué medida se cumple como tal, sin embargo, permite evaluar de manera general el acatamiento de disposiciones legales en cuanto al manejo de la información como lo expresa la LOTAIP y las estrategias utilizadas para una mejor y mayor difusión de los servicios. En este sentido, el GADM Chone cumple con el 78,95\% en lo referente al cumplimiento de indicadores, tal como se aprecia en la Tabla 6.

Tabla 6. Proporción del cumplimiento de los criterios de evaluación de la página web del GADM Chone

\begin{tabular}{|l|c|c|c|c|}
\hline Criterios sección contenido & Total de Items & Cumplimiento & Porcentaje & Porcentaje total \\
\hline 3. Información & 16 & 15 & $93,75 \%$ & $39,47 \%$ \\
\hline 4. Servicios y productos & 12 & 11 & $91,67 \%$ & $28,95 \%$ \\
\hline 5. Consultas y trámites & 10 & 4 & $40,00 \%$ & $10,53 \%$ \\
\hline \multicolumn{1}{|c|}{ Total } & 38 & 30 & & $78,95 \%$ \\
\hline
\end{tabular}

Para establecer la evaluación a la calidad se sumaron las ponderaciones obtenidas por cada criterio, estableciendo en un 78,98\% la calidad de la página Web del GAD Municipal del cantón Chone, como se establece en la Tabla 7 a continuación. 
Tabla 7. Evaluación final de la calidad de la página web del GADM Chone

\begin{tabular}{|l|c|c|c|c|c|c|}
\hline \multicolumn{1}{|c|}{$\begin{array}{c}\text { VARIABLES DE } \\
\text { ESTUDIO }\end{array}$} & Indicador & Calificación & $\%$ & $\begin{array}{c}\text { Aporte a la } \\
\text { ponderación } \\
\text { total }\end{array}$ & $\begin{array}{c}\text { Aporte real } \\
\text { a la } \\
\text { ponderación } \\
\text { total }\end{array}$ & $\begin{array}{c}\text { Calificación } \\
\text { de calidad }\end{array}$ \\
\hline $\begin{array}{l}\text { 1. Diseño de la } \\
\text { página web del GAD } \\
\text { Municipal de Chone }\end{array}$ & 40 & 34 & $85,0 \%$ & $15 \%$ & $12,73 \%$ & \\
\cline { 1 - 5 } $\begin{array}{l}\text { 2. Accesibilidad de la } \\
\text { página web del GAD } \\
\text { Municipal de Chone }\end{array}$ & 45 & 38 & $84,4 \%$ & $15 \%$ & $12,67 \%$ & \multirow{2}{*}{$\mathbf{7 8 , 9 8 \%}$} \\
\cline { 1 - 5 } 3. Información & 80 & 73 & $91,3 \%$ & $30 \%$ & $27,34 \%$ & \\
\hline $\begin{array}{l}\text { 4. Servicios y } \\
\text { productos }\end{array}$ & 60 & 50 & $83,3 \%$ & $25 \%$ & $20,83 \%$ & \\
\hline $\begin{array}{l}\text { 5. Consultas y } \\
\text { trámites }\end{array}$ & 50 & 18 & $36,0 \%$ & $15 \%$ & $5,41 \%$ & \\
\hline \multicolumn{1}{|c|}{ Total } & 275 & 213 & & $100 \%$ & $\mathbf{7 8 , 9 8 \%}$ & \\
\hline
\end{tabular}

\section{DISCUSIÓN}

Las variables de estudio de la presente investigación, tenían como objetivo establecer el nivel de calidad de los servicios en línea ofertados por el GADM Chone, a través de su página web. Para el efecto, partimos de los antecedentes históricos de los servicios en línea presentes en el mundo desde hace un poco más de diez años, según plantea Jarrín (2017) quien destaca que la tecnología es una herramienta de gran alcance, un canal a través del cual se puede ofrecer un servicio en condiciones favorables para el usuario y para la organización que lo ofrece.

Para determinar las dimensiones de las variables de estudio, se trabajó con lo que disponen los marcos normativos correspondientes y con el criterio de expertos en esta temática con aval académico y científico, acuerdo a lo que plantean las empresas Web consultadas, mismas que fueron PentaWeb, Incdustry y Masdigital. Estos portales coinciden en tres elementos para análisis, siendo éstos el diseño, accesibilidad y contenido, y cada una de estas variables cuenta con criterios específicos para su calificación.

En función de estas características, se desarrolló este estudio, y de acuerdo con los hallazgos de la investigación, la suma de las las ponderaciones obtenidas por cada criterio, establecieron en un 78,98\% del cumplimiento de la calidad del servicio de la página web del GADM Chone.

\section{CONCLUSIONES}

Este estudio tuvo como objetivo valorar la calidad de los servicios en línea del GADM Chone. Por tal motivo, se analizó la página web y se contemplaron tres criterios de evaluación que fueron diseño, accesibilidad, contenido. Este último se lo dividió en otros tres criterios que fueron información, servicios y productos, y consultas y trámites.

El parámetro de diseño, se evidenció que la página web del GADM Chone cumple con todos los ítems investigados y las valoraciones que tributa un $15 \%$ al resultado global, arrojó como valoración $85 \%$ de cumplimiento, destacándose el aspecto dinámico del portal. La accesibilidad consideró nueve 
parámetros de valoración y su resultado representa el aporte del 15\% del global, donde se obtuvo un $84 \%$ considerando que el portal permite el acceso desde dispositivos portátiles o computadores.

La sección de información de la página tiene 16 items valorados y su resultado parcial representa el 30\% del global. En este aspecto, los hallazgos positivos arrojan un 91\% de cumplimiento, especialmente en lo que respecta la información mandataria de la LOTAIP respecto a la rendición de cuentas de las autoridades. En la sección de servicios ofertados, existen 12 items evaluados de los cuales se cumplen 11, es decir que hay un $83 \%$ de cumplimiento, en donde además está el requerimiento de los usuarios, ya que comprende pagos en línea, agendamiento, entre otros. El segmento de consultas y trámites representan el aspecto con mayor deficiencia del portal, ya que no se evidencia guía para proveedores y contratistas en lo relacionado a servicios de proveedurías, o el seguimiento al avance de un trámite solicitado a través de esta modalidad, el alcance de esta valoración fue del $36 \%$, finalmente, se establece la calidad de la página Web del GAD Municipal del cantón Chone en 78,98\% en base a los parámetros calificados.

El presente estudio beneficia tanto al GADM Chone como a sus usuarios. El GADM Chone puede usar este estudio como un medio de verificación de la evaluación que tienen

sus servicios en línea, sobre todo enfocándose en aquellos componentes que deben ser mejorados. Mientras que, a nivel de usuarios se puede captar el interés de otros ciudadanos que se convertirían en nuevos usuarios, al conocer las ventajas de acceder a los servicios municipales desde dispositivos electrónicos.

\section{Bibliografía}

Asamblea, N. (2010). https://amevirtual.gob.ec/. Obtenido de https://bit.ly/32bshK2

Berners-Le, T. (06 de Junio de 2019). World Wide Web. New York: W3C. Obtenido de Introducción a la Accesibilidad Web.

C. Zambrano \& X. Vélez \& Y. Vélez . (2019). Ranking de gobierno electrónico en los GAD provinciales del Ecuador y municipales de Manabí . Dominio de la Ciencia, 356 - 374

Congreso, N. (2004). Ley Orgánica de Transparencia y Acceso a la Información Pública

Obtenido de Registro Oficial Suplemento 337: https://bit.ly/3iTEOsi

Díaz, I. (2018). Análisis de la funcionalidad del portal web del GAD Las Naves de la provincia de Bolívar. Babahoyo: Repositorio de la Universidad de Babahoyo.

GADM Chone. (09 de 0 9 de 2020). GADM Chone. Obtenido d e https://www.chone.gob.ec/

Hernández, J. (2018). La importancia del diseño web de calidad. Marketaria, 67-70. Incdustry. (14 de 02 de 2019). 5 Secciones que no pueden faltar en tu página web.

Obtenido de https://bit.ly/2Eqe6J6

J. Herrera \& M. Navia. (2020). Las tecnologías de la información: aliado y soporte para las organizaciones. RISTI:Revista Ibérica de Sistemas e Tecnologias de Informaçión, 11 - 12.

Jarrín, D. (2017). Percepción entre la calidad de servicio en línea y la satisfacción en la experiencia de usuarios corporativos de sitios web de viajes en la ciudad de Quito. Dialnet, 47 - 60.

Líppez-De Castro, S., \& García Alonso, R. (2016). Ciudadanos y gobierno electrónico: la orientación al ciudadano de los sitios Web municipales en Colombia para la promoción de la participación. Scielo, Universitas Humanística, 279-304.

M. Sánchez y A. Villarejo . (2004). La calidad de servicio electrónico: un análisis de los efectos moderadores del comportamiento de uso de la web. Sevilla : Depósito de la Universidad de Sevilla.

Martín-Díaz, M. (2012). Factores determinantes de la innovación tecnológica para las empresas pequeñas. Dialnet, 146 - 153.

Masdigital. (03 de 08 de 2016). Obtenido de Las 6 secciones esenciales de una página web: https:// bit.ly/2CKOSEC 
Merlo, A. (2013). La evaluación de la calidad de la información web: aportaciones teóricas y experiencias prácticas. Recursos informativos: Universidad de Salamanca, 101 - 110.

Paladines, X. (2015). Análisis, diseño e implementación de un sitio web dinámico para el GAD parroquial de Virgen de Fátima. Guayaquil: Repositorio de la Universidad Católica Santiago de Guayaquil.

PentaWeb. (22 de 02 de 2017 ). Partes de una página web . Obtenido de https://bit.ly/2E3jJ00
Sanahuja, J. (2020). Aliados de la gobernanza global. Anuario Ceipaz, 27 - 54. Schumpeter, J. (1978). Teoría del desenvolvimiento económico. México: Fondo de Cultura Económica (Quinta reimpresión).

Zambrano Paladines, M., Alejo Machado, O. J., \& Zambrano Proaño, P. (2019). Factores influyentes en la lealtad electrónica hacia comunidades turísticas en línea: Caso mochilero . Revista de Ciencias Sociales, 200-217. (c) $($ ) $(2)$

CREATIVE COMMONS RECONOCIMIENTO-NOCOMERCIAL-COMPARTIRIGUAL 4.0 .

\section{CitAR ESTE ARTICULO:}

Zambrano Zambrano, M. J., \& Rivadeneira Barreiro, L. (2021). Calidad de los servicios en línea del GADM Chone. RECIMUNDO, 4(4), 548-562. https://doi. org/10.26820/recimundo/4.(4).octubre.2020.548-562 